\title{
Recommendation of RILEM TC 212-ACD: acoustic emission and related NDE techniques for crack detection and damage evaluation in concrete*
}

\author{
Measurement method for acoustic emission signals in concrete
}

\author{
RILEM Technical Committee (Masayasu Ohtsu)**
}

Published online: 29 July 2010

(C) RILEM 2010

\section{Scope}

Acoustic emission (AE) is an inspection technique and one of nondestructive evaluation (NDE) methods

\footnotetext{
*The text presented hereafter is a draft for general consideration.

Comments should be sent within 6 months of publication to the TC chairman, Prof. Masayasu Ohtsu, Graduate School of Science and Technology, Kumamoto University, 2-39-1 Kurokami, Kumamoto 860-8555, Japan. Phone: +81-96-342-3542, Fax: +81-96-3423507, E-mail: Ohtsu@gpo.kumamoto-u.ac.jp.
}

**This recommendation was developed by a work group within RILEM TC212-ACD under the leadership of Mr. Masayasu Ohtsu, Japan.

\section{TC-Membership:}

Chairman: Masayasu Ohtsu, Japan.

Secretary: Tomoki Shiotani, Japan.

Members: M. Shigeishi, T. Kamada, S. Yuyama, T. Watanabe, T. Suzuki, Japan; J.G.M. van Mier, T. Vogel, Switzerland; C. Grosse, R. Helmerich, Germany; M.C. Forde, U.K.; A. Moczko, Poland; D. Breysse, France; S.A. Ivanovich; Russia; A. Sajna, Slovenia; D. Aggelis, Greece; G. Lacidogna; Italy.

RILEM Technical Commitee 212-ACD

(Masayasu Ohtsu) ( $\square)$

Graduate School of Science and Technology, Kumamoto University, 2-39-1 Kurokami, Kumamoto 860-8555, Japan e-mail: Ohtsu@gpo.kumamoto-u.ac.jp. by means of detecting elastic waves due to dynamic motions at $\mathrm{AE}$ sources, such as cracking, delamination, cleavage, and fretting in a material. The technique is widely applied to a variety of construction materials and basics for research are established [1]. Concrete structures could deteriorate due to heavy traffic loads, fatigue, chemical reactions, unpredictable disasters, and poor workmanship, although concrete structures have long been referred to as maintenance-free. Eventually, retrofit and rehabilitation of the structures are in heavy demand all over the world. It results in the need for the development of advanced and effective inspection techniques prior to repair works. In this regard, AE techniques have been extensively studied in concrete engineering. These are inspection techniques by means of detecting elastic waves due to cracking and damage (micro-crack) accumulation, originally developed in materials science, seismology, and mechanical engineering.

Focussing on crack detection and damage evaluation, it is known that $\mathrm{AE}$ techniques are prospectively applicable to concrete and concrete structures. This is because AE events or phenomena are to be observed under in-service conditions. Here, a measurement method is standardized for detecting $\mathrm{AE}$ signals in concrete.

This recommendation is referred to several standards established or published in the references [2-18], of which the newest version shall be cited. 


\section{Definitions of technical terms}

(a) $A E$ : transient elastic waves generated by the release of energy within a material. Microscopic fracture in concrete takes place with the release of stored strain energy as nucleating microcracks and generating elastic waves. These waves due to crack nucleation are referred to as $\mathrm{AE}$ waves, which propagate inside a material and are detected by an AE sensor as shown in Fig. 1.

(b) AE signal: The electrical signal detected at a sensor, which is converted through the detection of $\mathrm{AE}$ wave (elastic wave).

(c) Burst emission: A description of the signal that has a rapid rise to peak and slower decay to the noise level. Burst-type AE signals are typically detected in concrete. In contrast, under plastic deformation in metal, emissions are observed continuously without the decay. This is normally named "continuous emission."

(d) Channel: One line of AE signal detected by $\mathrm{AE}$ sensor and processed by the other devices.

(e) Hit: A hit is the term to indicate that a given $\mathrm{AE}$ channel has detected and processed one $\mathrm{AE}$ transient signal. Counting methods of AE signals are later described in the Sect. 4 of "signal analysis and AE parameters."

(f) Event: $\mathrm{AE}$ wave can be detected in the form of hits on one or more channels. One event is a group of $\mathrm{AE}$ hits received from a single source

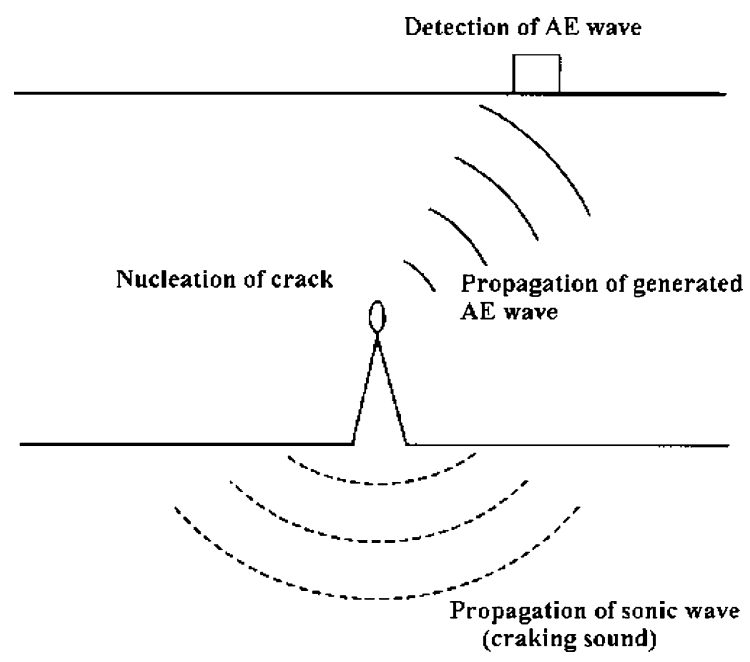

Fig. 1 Detection of AE waves by two or more channels, of which spatial coordinates could be located.

(g) Array: Spatial arrangement of AE sensors for spatially locating AE sources.

(h) Attenuation: The observed loss of a signal as it travels through a medium.

(i) Noises: Signals produced by causes other than $\mathrm{AE}$ phenomena. Elimination of noises is essential for effective detection of $\mathrm{AE}$ signals.

\section{Measuring system}

AE measuring system shall consist of the following devices with prescribed functions. A basic system is illustrated in Fig. 2, where only analog devices are shown. Following this system, a digital signal-processor is usually equipped.

\subsection{Sensor}

AE sensors shall be sensitive enough to detect $\mathrm{AE}$ signals generated in the target structure, taking acoustic coupling into consideration. They convert elastic waves (motions) on the surface of a material into electric signals, preferably, without any distortions.

A resonance-type sensor is most sensitive around the resonant frequency, while a broad-band sensor has approximately flat response in the range but is less sensitive than the resonance-type. So far, resonant frequencies of resonance-type sensors have been applied to concrete in the range of $50-250 \mathrm{kHz}$. AE sensor shall be robust enough against temperature change, moisture condition and mechanical vibrations in the environments.

Sensitivity calibration of AE sensors shall be performed by employing the standard source. A simulated $\mathrm{AE}$ source due to pencil-lead break has been defined [8, 11, 16]. This standard source is illustrated in Fig. 3, where a guide ring is recommended to be employed. The pencil-lead break is known as Hsu-Nielsen source in AE technology $[19,20]$.

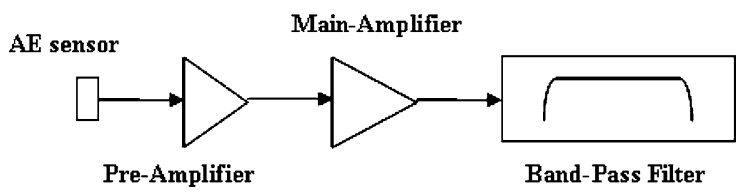

Fig. 2 AE measuring system 


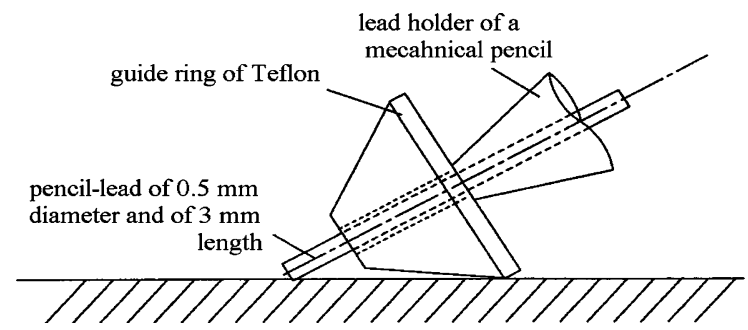

Fig. 3 AE standard source by pencil-lead break

\subsection{Amplifier}

Amplifiers normally consist of the pre-amplifier and the main amplifier as shown in Fig. 2. The preamplifier shall be located as close as possible to $\mathrm{AE}$ sensor. The internal noise of the amplifier shall be inherently low and less than $20 \mu \mathrm{V}(26 \mathrm{~dB}$ for $0 \mathrm{~dB}=1 \mu \mathrm{V})$ as the peak voltage converted by input voltage. Here, the gain of the amplifier is given in $\mathrm{dB}$ (decibels), which means the ratio of the output voltage $V_{\mathrm{o}}$ to the input voltage $V_{\mathrm{i}}$ as,

$\mathrm{dB}=20 \log _{10}\left(V_{\mathrm{o}} / V_{\mathrm{i}}\right)$

A total gain of a pre-amplifier and a main amp. employed in concrete is $40-60 \mathrm{~dB}$. Again, the amplifiers shall be robust enough against the environmental conditions and be protected properly.

\subsection{Filter}

The frequency range shall be determined prior to the measurement, taking into account the performance of $\mathrm{AE}$ sensors and the amplifiers. Selection of the frequency range is closely related to elimination of noises. In concrete, a band-pass filter between around $10 \mathrm{kHz}$ and several $100 \mathrm{kHz}$ up to $3 \mathrm{MHz}$ is employed.

\section{Signal analysis and $\mathrm{AE}$ parameters}

One waveform is to be counted as one AE hit, while the cycles over the threshold level are named as AE ring-down counts (or simply "counts"). Here, the threshold is a preset voltage level, which has to be exceeded before one $\mathrm{AE}$ signal is detected and processed. The threshold level applied in AE measurement is $30-50 \mathrm{~dB}$ in concrete.

Methods for AE counting are illustrated in Fig. 4. In the case of multi-channel observation, $\mathrm{AE}$ occurrence is monitored at each sensor location. At some sensors, AE signals may not be observed due to attenuation or undetectable propagation path. The number of the events which are counted at one of the channels corresponds to the number of $\mathrm{AE}$ hits defined above.

In addition to $\mathrm{AE}$ count, $\mathrm{AE}$ hit and $\mathrm{AE}$ event, the following $\mathrm{AE}$ parameters shall be obtained by the measurement system.

(1) Peak amplitude

(2) AE energy

(3) Rise time

(4) Duration time

(5) Arrival time differences in AE sensor array

(6) External parameters: The measurement system shall be able to obtain time information along with AE parameters. In addition, such external parameters as load, strain and so forth are preferably recorded in the system.

The waveform parameters of arrival time, duration and peak amplitude are displayed in Fig. 5, showing the threshold level. The rise time means a duration from the arrival to the peak. There exist a variety of definitions on AE energy. In principle, AE energy corresponds to an area of the envelope of the waveform.

\section{Setup and measurement}

\subsection{Sensor setup}

AE sensors shall be calibrated properly in advance of the measurement. They are attached at proper locations to cover the target area. The period of the measurement shall be prescribed, depending on the following conditions:

(a) Propagation property of AE signals in the target structure

(b) Stress distribution in the structure under inspection

\subsection{Environmental noises}

In advance to $\mathrm{AE}$ measurement, the noise level shall be estimated. Then, counteract against external noises, wind, rain, sunshine and so forth shall be conducted to decrease the noise level as low as possible. In the case that the noises have similar 
Fig. 4 AE counting methods. a Method for AE counts. b AE event or hit counted by pulse. c AE event or hit counted by envelope
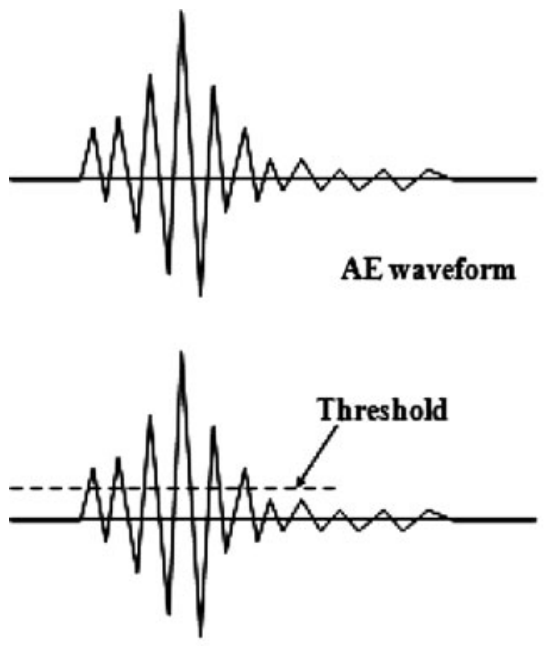

Ringdown-counting

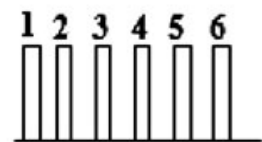

(a)

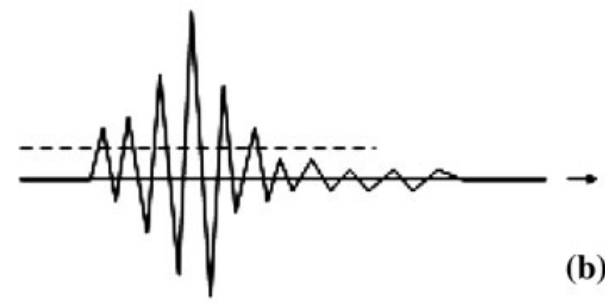

Event counting (hits)

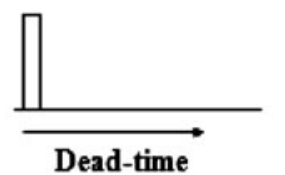

(b)
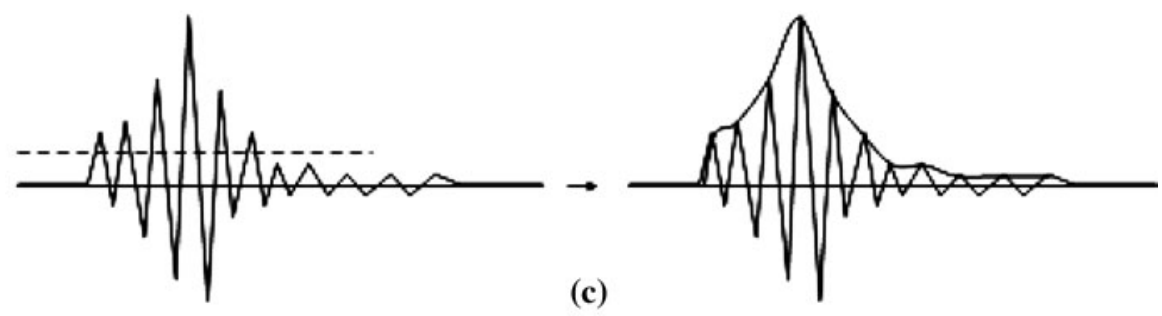

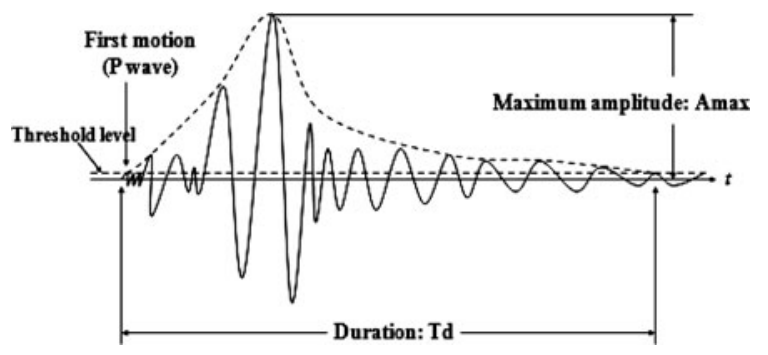

Fig. 5 AE waveform parameters

frequency contents, amplitudes to $\mathrm{AE}$ signals or sources of the noises are unknown, characteristics of the noises shall be estimated prior to the measurement. Based on this result, separation of AE signals from the noises shall be achieved. In this respect, the use of filters is applicable after determining the proper frequency range.

\subsection{Measurement}

In an existing structure, a measurement is normally conducted under loads which must not make a critical damage on functions of the structure to detect and locate active cracks. Based on the spatial area to be covered by AE sensors, those of proper frequency characteristics shall be selected. In advance to the test, attenuation properties of the target structure shall 
be estimated, by employing the standard source or the equivalent.

Based on this information, sensor array shall be determined so as to keep the equivalent sensitivities in all the sensors. AE signals due to cracking shall be detected properly for the duration of the measurement. Concerning AE parameters detected, their trend, distribution, correlation, and locations are monitored. In addition, multi-channel observation provides to locate $\mathrm{AE}$ sources, by applying the location routine available.

\subsection{System inspection}

Sensitivity of AE channels shall be conducted routinely by employing the standard source. Variation within the channels shall be less than $3 \%$ in the voltage.

\subsection{Storage of data}

The system shall be equipped with an enough memory to record the data measured. It is preferable that all the data recorded are analyzed digitally by computer.

\section{Documents}

Documents to report the results shall contain the following articles:
(a) Date
(b) Test person
(c) Devices
(d) Measurement locations
(e) Results of system inspection before and after the setup
(f) Results of analytical data before and after the setup

\section{References}

1. Grosse CU, Ohtsu M (eds) (2008) Acoustic emission testing. Springer, Heidelberg

2. EN (European Norms) 1330-9 Nondestructive testingterminology_Part 9: terms used in AE testing

3. EN 13554 Nondestructive testing-acoustic emissiongeneral principles

4. EN 13477-1 Nondestructive testing-acoustic emissionequipment characterization-Part 1: equipment description

5. EN 13477-2 Nondestructive testing-acoustic emissionequipment characterization-Part 2: verification of operating characteristics

6. ASTM (American Society for Testing and Materials) E1316 Standard definitions of terms relating to AE

7. ASTM E650 Standard guide for mounting piezoelectric AE sensors

8. ASTM E750 Standard practice for characterizing AE instrumentation

9. ASTM E976 Standard practice for determining the reproducibility of AE sensor response

10. ASTM E1106 Standard method for primary calibration of AE sensors

11. ASNT (American Society for Nondestructive Testing) DGZfP-SE1 Nondestructive testing: acoustic emission terms

12. ASNT DGZfP-SE2 Guide line for AE sensor calibration

13. ASNT DGZfP-SE3 Guide line for AE characterization during AE test

14. AFNOR (French Association for Standards) NF A09-350 Nondestructive testing-vocabulary used in acoustic emission

15. EWGAE (European Working Group on Acoustic Emission) Codes for AE examination: Code I-location of discrete acoustic events

16. EWGAE codes for AE examination: Code IV—definition of terms in $\mathrm{AE}$

17. JSNDI (Japanese Society for Nondestructive Inspection) NDIS 2110 calibration of AE sensors

18. NDIS 2421 Recommendation practice for in situ monitoring $\mathrm{AE}$ of concrete structures by $\mathrm{AE}$

19. Hsu NN, Breckenridge FR (1981) Characterization and calibration of acoustic emission sensors. Mater Eval 39:60-68

20. Nielsen A (1977) Acoustic emission source based on pencil lead breaking. Paper presented at EWGAE meeting in Rome, 21 Sept 